\title{
Erratum to: On the reduction of gloss property of organic coated sheet metals after forming
}

\author{
H.-D. Pham - M. Trompeter $\cdot$ A. E. Tekkaya
}

Published online: 28 August 2010

(c) German Academic Society for Production Engineering (WGP) 2010

\begin{abstract}
Organic coated sheet metals (OCSM) are widely used in many industrial applications such as automobile or electrical appliances because of their corrosion resistance and satisfaction of ecological requirements. Nevertheless, original functions of the very thin coating layer in OCSM products may be altered after being formed which consequently leads to a reduction of the product's quality. This paper deals with the reduction of gloss property of OCSM products. A forming limit diagram (FLD), a forming limit diagram of the coating layer (FLDC) of OCSM and a forming diagram for the prediction of gloss reduction are determined by using Nakajima tests. The Nakajima tests are performed with predefined strain states of the specimen. The experimental results show the dependency of the FLDC curve and the gloss reduction on both the equivalent strain and the strain ratio. Deep-drawing tests prove the applicability of the determined forming diagrams to predict coating fracture and gloss properties of OCSM after sheet metal forming and, thus, their potential as a tool for the process design for OCSM products.
\end{abstract}

\footnotetext{
"Unfortunately, a previous version of this paper which had not been authorized by the co-authors was published on http://www.springerlink.com on 15th February 2010. The present paper is jointly prepared but deviates from the previous version concerning the presentation as well as the interpretation of the results".
}

The online version of the original article can be found under doi:10.1007/s11740-010-0215-8.

H.-D. Pham $(\bowtie) \cdot$ M. Trompeter · A. E. Tekkaya

Institute of Forming Technology and Lightweight Construction,

Baroper Straße 301, 44227 Dortmund, Germany

e-mail: Ha-Duong.Pham@tu-dortmund.de
Keywords Organic coated sheet metal - Surface quality · Forming limit diagram

\section{Introduction}

Organic coated sheet metals are used in various industrial sectors, from beverage can manufacturing to automotive field, because of their powerful functions such as corrosion resistance property, environment-friendliness and satisfaction of ecological requirements. In civil engineering, for instance, these products are often used for wall cladding and roofing. In the domestic appliance market, they are dominantly used for household items like refrigerators, washing machines, and for smaller kitchen appliances such as microwave ovens or anti-sticky pans, etc. By utilizing this material, the number of necessary manufacturing steps as well as production cost is significantly reduced while the product quality is importantly improved [1, 7].

Basically, organic coated steel is composed of a steel substrate (cold rolled or with a zinc-based metallic coating) with a surface treatment layer, a paint prime and a topcoat, see Fig. 1.

The thickness of the organic coating layers is very thin compared to the thickness of the steel substrate, and the ratio often ranges from 1:2.5 to 1:50. Furthermore, organic coated materials and steel substrates greatly differ in their mechanical behavior, especially when these materials are in large deformation states. Basically, the very thin coating layer is extremely "sensitive" to the forming conditions and this is the major reason which unexpectedly leads to failures of the product such as gloss reduction, color deviation and crazing [2]. The typical failure models are depicted as shown in Fig. 2. 


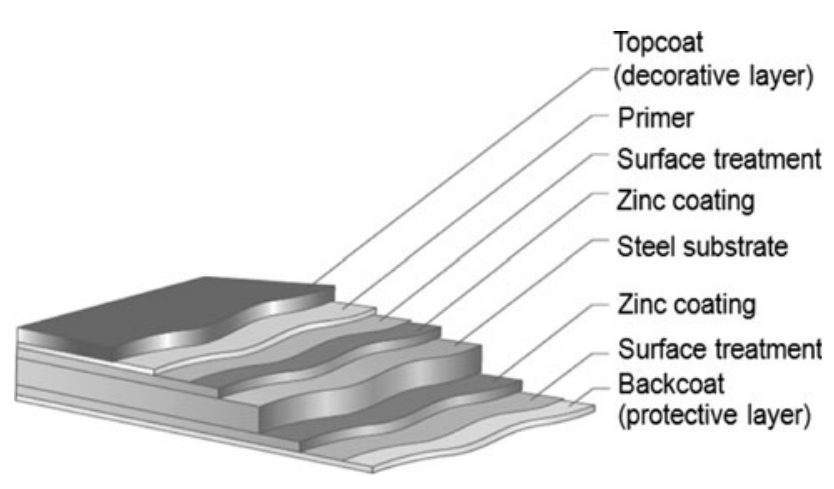

Fig. 1 General construction of OCSM [1]

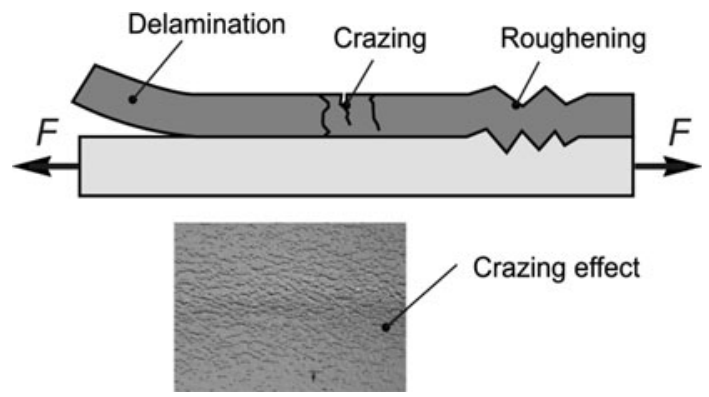

Fig. 2 Typical failures of coating layer [2]

A crucial requirement of forming technology is to maintain all the original functionalities of the coating layer e.g. optical and corrosion property after industrial forming processes. That means the undesirable effects such as loss of gloss, crazing or color deviation must be predicted based on a suitable failure criterion.

Various researchers have presented results concerning the forming of OCSM so far [2-6, 9, 11-14, 19, 20]. However, most researchers have just focused on corrosion protection, delamination phenomenon or cohesive zone of OCSM. Nevertheless, less attention is dedicated to the change of optical properties of products. Some recent contributions have solved this problem with the forming limit curve. For example, Kleiner [2] developed forming diagrams that allow a prediction of the change of surface properties of the coating layer based on the strain states of steel substrate. The major limitation of this research is the assumption that the coated and the substrate material have identical strain states. But in fact, they are not similar due to the difference of mechanical behavior.

This paper investigates the change of gloss property depending on the strain states of the OCSM products. The methodology is carried out in sequence as following. The experimentally characterized forming limit diagram of the base material (FLD) and the forming limit diagram of the coating layer (FLDC) are first used to describe the formability and fracture limit of OCSM. Subsequently, a series of Nakajima tests with predefined strain value are performed to characterize the loss of gloss on the coated surface. The strain states corresponding to the loss of gloss on specimens are also measured by using an optical measuring system, namely GOM-Argus. Finally, by combining the FLD, the FLDC and gloss reduction diagrams are determined, which can be applied for the design of sheet metal forming processes.

\section{Experimentation}

\subsection{Experimental set-up}

The Nakajima tests were first performed on an Erichsen universal testing machine at room temperature to provide data for both FLD and FLDC diagrams. According to the Nakajima test, a hemispherical punch with a diameter of $50 \mathrm{~mm}$ and various OCSM specimens with identical diameters of $100 \mathrm{~mm}$ but different groove radii $(R=0,20$, $25,30,35,40,45 \mathrm{~mm}$ ) were employed to investigate the different states of stress and strain, see Fig. 3a [18]. For statistical reasons, five specimens on each groove radius were used.

Due to the difference in mechanical behavior between the coated and the substrate material, the strain state and the fracture mechanism are also different. Consequently, the procedure for establishing the FLD and FLDC is also not in a similar way. For the determination of the FLD, these specimens were stretched until necking occurred on the steel substrate. For the determination of the FLDC, all the specimens were only stretched until cracks occurred on the coating layer, Fig. $3 \mathrm{~b}$.

The strain state of the specimens is analyzed by using an optical measuring system that allows a strain measurement on deformed parts, see Fig. 3c. By using the maximum of the measured major logarithmic strain $\varphi_{1}$ and the minor logarithmic strain $\varphi_{2}$ at fractured area, the limiting curves, namely FLD and FLDC, are plotted for every specimen. The final result was averaged by using the data from five specimens. These curves represent the border between safe and failure region. The strain states below the determined curves act as process window for the whole forming process.

\subsection{Material}

The investigated material was coil-coated steel sheet on both sides, i.e. a decorative coating layer on the upper surface and a protective layer on the other one. For the steel substrate, DC04 steel with a thickness of $0.8 \mathrm{~mm}$ was used because of its excellent cold-formability. The mechanical properties of the substrate material are summarized in 
Table 1. Polyurethane (PUR) was used as decorative film with a thickness of $0.03 \mathrm{~mm}$. The characterizations of PUR were tabulated in Table 2 .

\subsection{Gloss measurement}

One of the most important criteria of the surface quality is gloss reduction. The term "gloss" is based on the interaction of light and surfaces of specimens depending on the physical characteristics of the surface. It is the reflection of
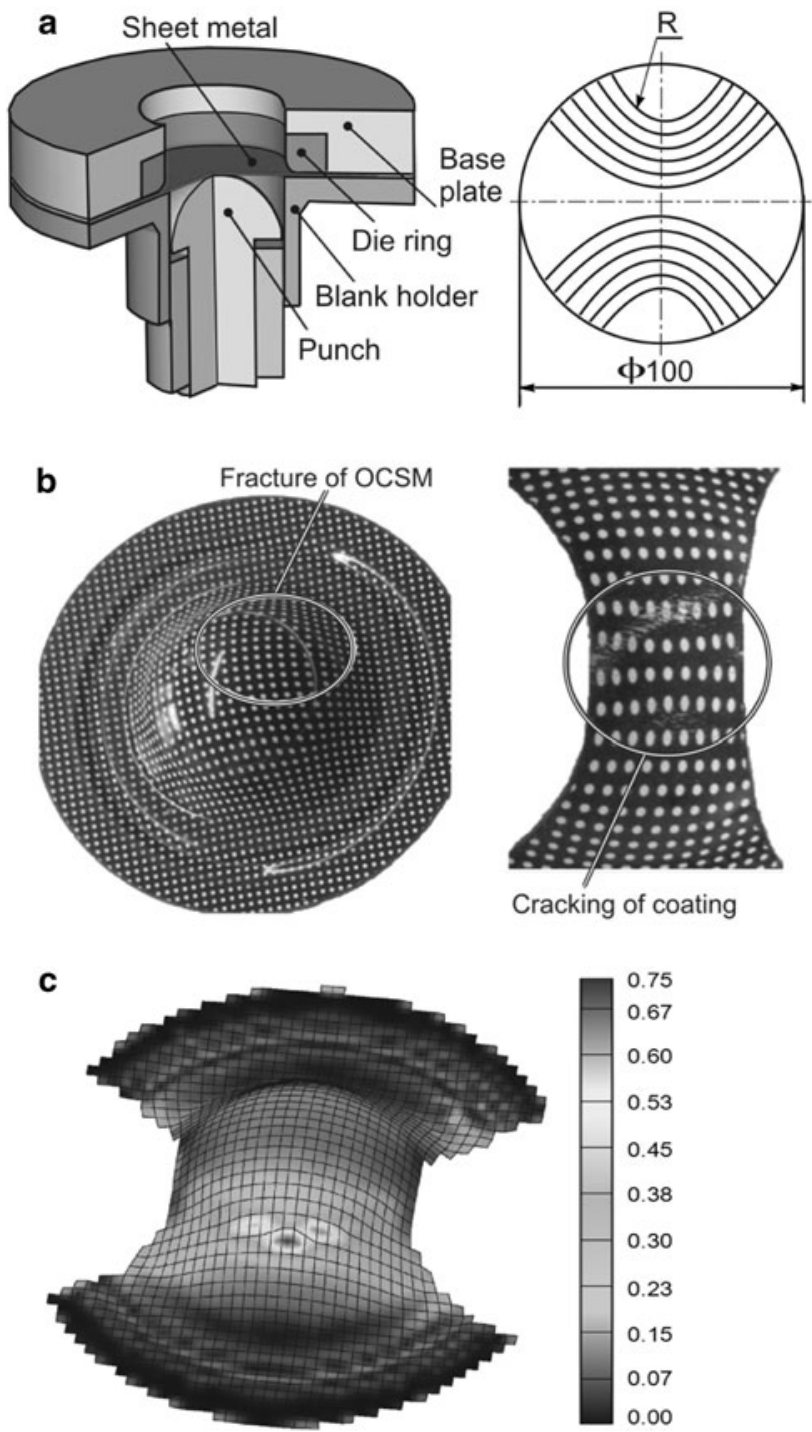

Fig. 3 Nakajima test for determination of the FLD and FLDC. a Experimental set-up and specimens. b Fracture of substrate and cracking of coating layer. c Optical strain measurement on specimen
Table 2 Mechanical properties of the coated material

\begin{tabular}{lll}
\hline Properties of PUR & Unit & Value \\
\hline Tensile strength & ISO 527, N/mm & $<40$ \\
Elongation at break & ISO 527, \% & $<700$ \\
Tensile Young's modulus & ISO 527, N/mm² & $<650$ \\
Rockwell-Hardness & Shore-Hard & $68-99 \mathrm{~A}$ \\
Surface hardness & & Good \\
Formability/bending & & Excellent \\
Formability/drawing & & Excellent \\
Colour & & Black \\
\hline
\end{tabular}

the light into specular direction when a certain light is radiated on the surface. The loss of gloss was analyzed by the following method described in DIN ISO 2813 [8]. According to this, the effect of alignment or elongation of the polymer chains of the coating and the resulting changes in the reflection characteristics was determined. The gloss reduction is measured by using a reflector meter. The principle of the gloss measurement is illustrated in Fig. 4.

The common irradiation angles of incidence for the gloss measurement are $20^{\circ}$ (high gloss), $60^{\circ}$ (medium gloss) and $85^{\circ}$ (flat gloss). For low gloss surfaces, the angle $85^{\circ}$ of incident is recommended [15]. Here, two types of gloss measure apparatuses are used: one for flat surfaces and the other one for curved surfaces.

To characterize the change of gloss property, the gloss value before being formed is measured and denoted by $G_{\mathrm{o}}$. The updated gloss value is measured afterwards and denoted by $G_{i}(i=1,2,3, \ldots)$. The deviation of these values is denoted by $\Delta G$ and computed as follows:

$\Delta G_{i}=G_{o}-G_{i}$

The loss of gloss (denoted by $L G$ ) is defined by the following equation:

$L G_{i}=100 \cdot \frac{\Delta G_{i}}{G_{o}}=100 \cdot \frac{G_{o}-G_{i}}{G_{o}}($ in $\%)$

According to equation (2), the maximum and minimum loss of gloss can be determined as follows:

$L G_{i \_ \text {min }}=100 \cdot \frac{G_{o}-G_{i \_ \text {max }}}{G_{o}}($ in $\%)$
$L G_{i \_ \text {max }}=100 \cdot \frac{G_{o}-G_{i \_ \text {min }}}{G_{o}}($ in $\%)$

In this research, two cases of the Nakajima test were performed (see Fig. 5):

Table 1 Mechanical properties of the steel substrate

\begin{tabular}{llllllllll}
\hline Steel substrate DC04 & Young's mod. kN/mm & Rp 0.2 N/mm & Rm N/mm & Ag\% & A\% & r0 & r45 & r90 \\
\hline & 183.50 & 174.11 & 308.13 & 26.85 & 41.76 & 1.81 & 1.23 & 2.13 \\
\hline
\end{tabular}




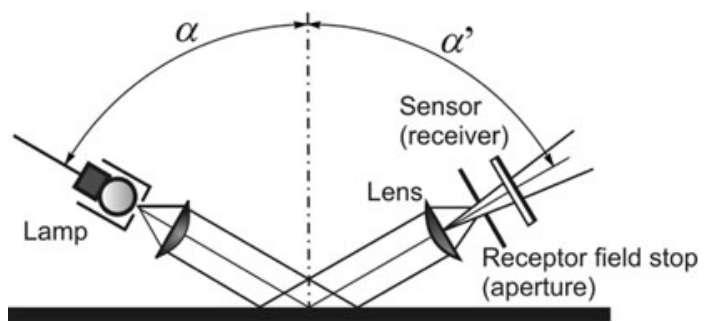

Fig. 4 Principle of gloss measurement

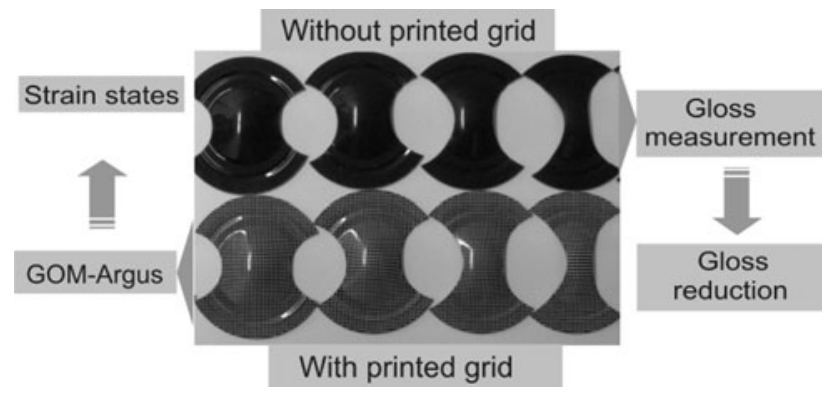

Fig. 5 Nakajima specimens for determination of the strain states and the gloss reduction

(a) specimens without printed grid on the surface to analyze the gloss reduction

(b) specimens with printed grid to analyze the strain states

\section{Results and discussions}

3.1 Forming limit diagram and fracture limit diagram of coating layer

The determined FLD and FLDC for the investigated material are represented in Fig. 6. The maximal strain value of the FLDC is lower than the one of the FLD, which means that the crazing effect occurs on the coating layer before the fracture appears on the steel substrate. Therefore, the FLDC should be used instead of the FLD in order to evaluate the formability of OCSM concerning the surface quality of the coating layer.

By using this diagram, the process limits at all relevant strain states which can occur in sheet metal forming, such as equibiaxial stretch-forming $\left(\varphi_{1}=\varphi_{2}\right)$, plane strain $\left(\varphi_{2}=0\right)$, uniaxial tension $\left(\varphi_{1}=-2 \varphi_{2}\right)$ and deep-drawing $\left(\varphi_{1}=-\varphi_{2}\right)$, are determined.

For the verification of this result, deep-drawing tests were performed. Before testing, the protective coating layer on one side of specimen was removed in order to evaluate the behavior of the steel substrate after forming. The obtained results show that the coating layer is cracked

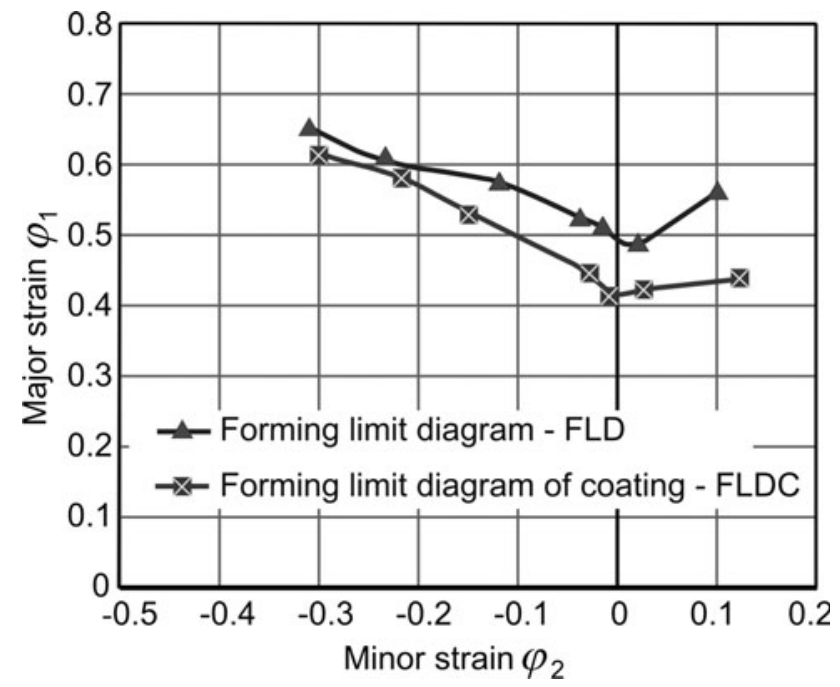

Fig. 6 FLD and FLDC of OCSM

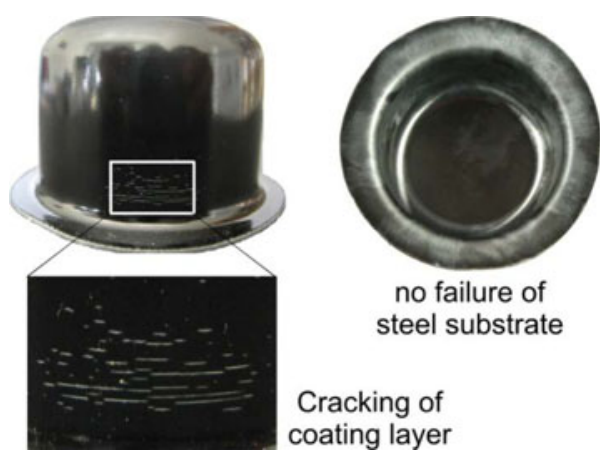

Fig. 7 Verification of the FLD and the FLDC

while the steel substrate is still intact, without failure, see Fig. 7.

In detail, the coating layer appears roughened at first, then crazed. The crazing effect is caused by ruptured molecular chains of the organic material. After crazing, delamination may happen if the deformation is continued increased. This phenomenon can be explained by the failure mechanism of organic coating layer on steel substrate. According to this, mechanical stresses imposed on the coated material and large plastic strains in the metal substrate will lead to coating failures. Among these induced failures, in-plane stresses are the causes of the defect of coated material itself i.e. crazing or roughening. Out-plane stresses are the reasons for losing adhesion and may lead to a debonding effect between two layers. On the other hand, large plastic strains in the substrate cause the loss of adhesion at the interface and may also lead to delamination in further processing, see [3, 9-12]. In addition, residual stresses in the coating resulting from plastic strains in the metal substrate may lead to an additional shear stress at the interface which is the cause of the severe defect of the 
coating layer [12]. In case of the specimen in Fig. 7, the defects of the coating layer were supposedly caused by the mechanical in-plane stress and the large plastic strains in the steel substrate.

\subsection{Gloss reduction versus strain states}

To evaluate the gloss reduction of the coating layer depending on the strain states, Nakajima tests with predefined depths have been performed. The respective strain states over specimens are measured and the corresponding gloss reductions are recorded. Figure 8a shows the measured strain values of the Nakajima tests. To allow comparisons, it is assumed that the strain value at the limited fracture of the coating layer corresponds to $100 \%$. The other values are 30,60 and 90\%, respectively. The gloss values are measured by using a reflectometer (NOVOCurve) under $85^{\circ}$ (flat gloss).

Figure $8 \mathrm{~b}$ shows the dependency of the gloss reduction on the equivalent strain and strain ratio. It can be observed that the loss of gloss increases with increasing equivalent strain $\bar{\varphi}$ as a consequence of the elongation of the polymer chains. The gloss value is reduced due to the strain depended surface roughness of the coating. However, the gloss reduction also depends on the strain ratio. Under biaxial tension and plane strain, the increase of the gloss reduction is much lower than in the compression-tension state.

Supposedly, this effect is a result of the occurrence of ripples on the metal substrate due to compressive strains. According to these results, large compressive strains must be avoided in order to maintain the origin gloss appearance of the coil-coated steel sheet. The results, which are shown in Fig. 8, can be used as reference data to predict the change of gloss properties after plastic deformation in sheet metal forming processes.

\section{Application}

Nowadays, numerous sheet metal forming processes are simulated by Finite Element Methods in order to ensure its feasibility and to optimize the tool geometry. In this regard, forming limit diagrams are widely used to determine at which deformation states the material will fail. The determined forming diagrams to predict coating fracture and gloss properties of OCSM can be used in a similar way in order to design sheet metal forming processes which are optimized regarding the processing of OCSM.

The applicability of these forming diagrams could be proved by deep drawing tests. A cylindrical cup was drawn by using OCSM with and without grid. The strain distribution over the gridded deep-drawing part was analyzed by
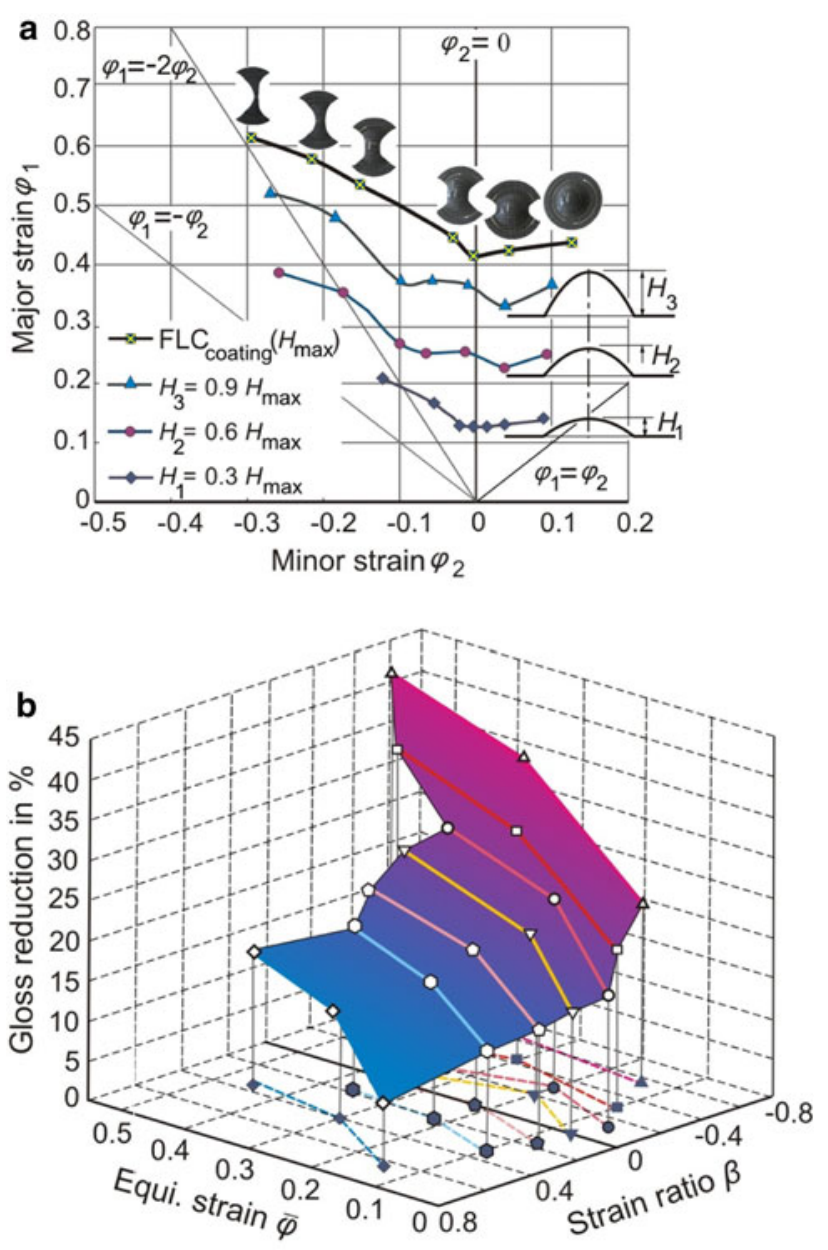

Fig. 8 Gloss reduction versus strain states. a Measured strains of the Nakajima specimens. b Gloss reduction depending on equivalent strain and strain ratio

using an optical strain measurement system (GOM-Argus). The corresponding gloss reduction in the area around these points was also measured by using a reflectometer (NOVOCurve).

Figure 9 shows the strain distribution over the formed part and the strain values at the corresponding points mentioned above.

The strain values at three characteristic points, which represent different strain states

- point at the bottom area (denoted by B)

- point at the punch radius (denoted by P)

- point at the side wall area (denoted by $\mathrm{S}$ )

are considered in detail and inserted into the diagram of Fig. 8 (see Fig. 10). The strain states at the points B and P match well the strain states of the Nakajima specimens and, hence, the gloss values can be compared without interpolation. Only the strain states at the points $C$ are beyond the strain states which could be attained by the Nakajima 


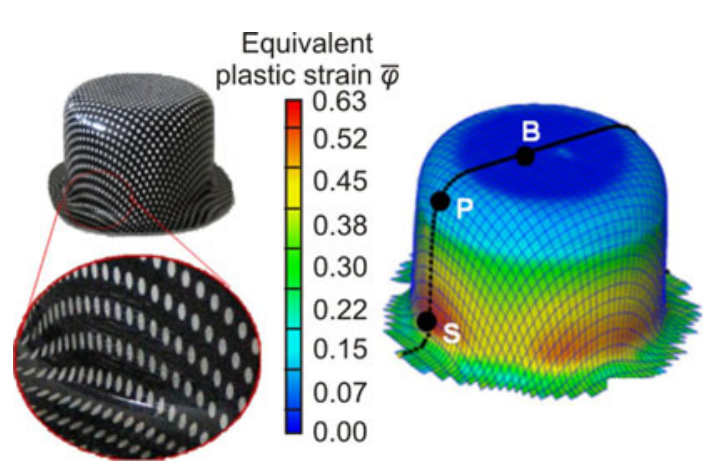

Fig. 9 Measured strain distribution of the deep-drawing part

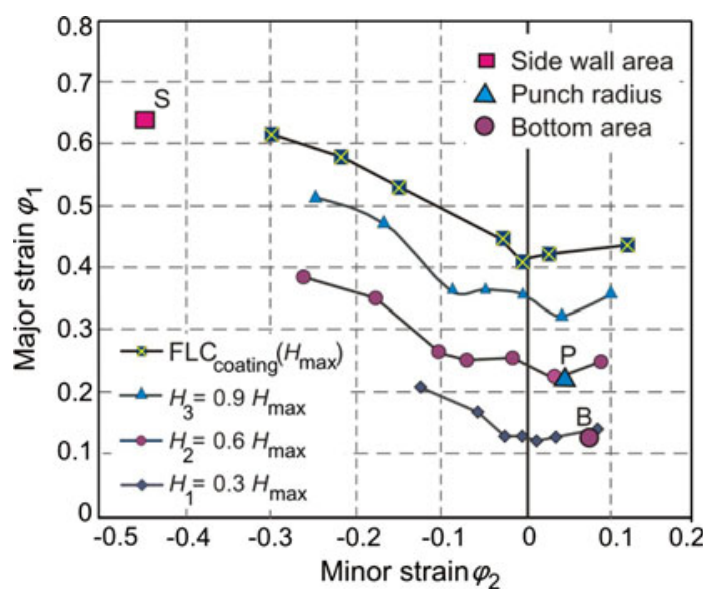

Fig. 10 Strain state at the points $B, P$, and $S$

specimens. As can be seen, the gloss reduction at the bottom area $\left(\varphi_{2}<0.1, \varphi_{1}>0.1\right)$ is only $L G_{\mathrm{B}}=5.2 \%$. Around the punch radius, where a higher deformation occurs $\left(\varphi_{2}<0.1,0.2<\varphi_{1}<0.3\right)$, the gloss reduction is considerably higher than the one at the bottom area and attains the value of $L G_{\mathrm{P}}=16 \%$. At the sidewall area, where the cracking phenomenon occurs on the coating layer, the gloss reduction is extremely high and attains the value of $L G_{\mathrm{S}}=69.2 \%$. This area shows the uniaxial tension state, i.e. $\varphi_{1}=-\varphi_{2}$ and exceeds the fracture limit of the coating layer, which is presented in Fig. 6. A comparison between the accuracy of the measured results obtained by the deepdrawing test and the reference results obtained by the Nakajima test is showed in Table 3. As computed in section 3.2 (see Fig. 8) the referred gloss reduction values are 7.7 and $18.56 \%$, while the correspondent values $L G_{\mathrm{B}}$ and $L G_{\mathrm{P}}$ of the deep-drawing part are 5.2 and $16 \%$, respectively. It is obviously that the deviation of the gloss values of the Nakajima specimens and the deep drawing part at assimilable states (point B and P) are between 2 and $3 \%$. Only at the point $\mathrm{S}$, an interpolation of the gloss values is required. The deviation between interpolated reference data and gloss values of the deep drawing part deviate about $3.30 \%$.
Table 3 Comparison of the gloss values of the Nakazima specimens (reference data) and the deep drawing part

\begin{tabular}{lll}
\hline & $\begin{array}{l}\text { Gloss reduction at deep } \\
\text { drawing part in \% }\end{array}$ & $\begin{array}{l}\text { Reference } \\
\text { data in } \%\end{array}$ \\
\hline B & 5.20 & 7.70 \\
P & 16.00 & 18.56 \\
C & 69.20 & 72.50 (failure by cracking) \\
\hline
\end{tabular}

These investigations demonstrate that the analyzed results of the deep-drawing part are in good agreement with the reference data obtained by the Nakajima test. As a result, the reference data can be applied to predict the optical property of OCSM products after industrial forming processes. According to the proposed method, it is necessary to establish gloss reduction curves versus different strain values. Then, based on these sample curves, the gloss reduction which corresponds to a specific strain value and strain state can be predicted.

\section{Conclusions}

This investigation focuses on the characterization of the gloss reduction of OCSM. The FLDC is established and can be used to define a suitable process window of the manufacturing sequence. The relation between the gloss reduction and the strain states is determined. According to this, the following conclusions are drawn:

- In order to evaluate the formability concerning the quality of the coating layer, the FLDC should be used instead of the FLD.

- An increase of the strain value leads to the increase of the loss of gloss.

- The strain ratio has a major influence on the gloss reduction. The loss of gloss always reaches the minimum value with the equibiaxial stretch state, at the same equivalent strain. The maximum gloss reduction occurs with the compression of the material $\left(\varphi_{2}<0\right)$.

- By combining the reference data and the FLDC, the gloss reduction after forming processes can be safely predicted.

Acknowledgments The authors would like to thank the German Academic Exchange Service (Deutscher Akademischer Austausch dienst - DAAD) for the funding of this research project.

\section{References}

1. Organic coated steel, User manual. www.arcelormittal.com

2. Hellinger V, Kleiner M, Schikora M (2002) Forming limit curve for the prognosis of the surface quality of Coil-Coated products, Research project KI 619/12-1, IUL, Tu. Dortmund 
3. Van der Bosch MJ (2007) Interfacial delamination in polymer coated metal sheet. Eindhoven University of Technology, The Netherlands

4. Van der Aa MAH, Schreuers PJG, Baaijens (2001) Modelling of the wall ironing process of polymer coated sheet metal. Mech Mater 33

5. Lawrenz K-J (1978) Untersuchungen zum Tiefziehverhalten organisch beschichteter Feinbleche und die Prüfung dieser Verbundwerkstoffe, Dr.-Ing. Dissertation, IUL, Universität Dortmund

6. Finckenstein Ev, Lawrenz KJ (1978) Umformen kunststoffbeschichteter Stahlbleche durch Tiefziehen mit starren Werkzeugen, Bänder Bleche Rohre

7. Jitsukawa M, Yamashita M (2003) Advanced coated steel sheets with excellent functions to satisfy ecological requirements, NKK Technical review, No. 88

8. DIN EN ISO 2813 (1999) Beschichtungsstoffe: Bestimmung des Reflektometerwertes von Beschichtungen unter $20^{\circ}, 60^{\circ}$ und $85^{\circ}$. Beuth-Verlag, Berlin

9. Vayeda R, Wang J (2007) Adhesion of coating to sheet metal under plastic deformation. Inter J Adh Adhes 27:480-492

10. Boelen B, Den Hartog H, Van der Weijde H (2004) Product performance of polymer coated packing steel, study of the mechanism of defect growth in cans. Prog Org Coat 50:40-46

11. Bastos AC, Ostwald C, Engl L, Grundmeier G, Simoes AM (2004) Formability of organic coatings- an electrochemical approach. Electrochim Acta 49:3947-3955
12. Polyakova A, Stepanov EV, Provder T, Hiltner A, Baer E (2000) Relationship of coating failure to deformation in the deep drawn cup. J Adh 72:1:37-50

13. Bernsprangb L, Hammam T (1993) Verification of an explicit finite-element code for the simulation of the forming process of rectangular box of coated sheet steels. J Mater Process Technol 39(34):431-453

14. Lang L, Danckert J, Nielsen KB (2005) Multi-layer sheet hydro forming: experimental and numerical investigation into the very thin layer in the middle. J Mater Process Technol 170:524-535

15. Smith KB (1999) Measuring the perception of glossy surfaces. Pigment Resin Technol 28:217-222

16. Bargel S (2005) Werkstoffkunde. Springer, New York, pp 385-405

17. MSC MARC, Nonlinear finite element analysis of elastomers, Technical Paper

18. Marciniak Z, Duncan JL, Hu SJ (2002) Mechanic of sheet metal forming, Butterworth-Heinemann, ISBN: 0750653000

19. Neugebauer R, Bräunlich H, Wolfhard M, Alsmann M (1998) Umformverhalten von bandbeschichteten Stahlfeinblechen. Stahlkolleg, Düsseldorf

20. Xia SM, Gao YF, Bower AF, Lev LC, Cheng Y-T (2007) Delamination mechanism maps for a strong elastic coating on an elastic-plastic substrate subjected to contact loading. Inter $\mathrm{J}$ Solids Struct 44:3685-3699 